\title{
A zero velocity intervals detection algorithm based on sensor fusion for indoor navigation systems
}

\author{
Ming $\mathrm{Ma}^{\dagger}$, Qian Song, Yang-Huan Li and Zhi-Min Zhou, \\ College of Electronic Science and Engineering, National University of Defense \\ Technology \\ Changsha, Hunan 410073, P. R. China \\ †E-mail: maming09@126.com
}

\begin{abstract}
The Zero-velocity Update (ZUPT)-aided Extended Kalman Filter(EKF) algorithm is commonly deployed to resolve trajectories of pedestrians. To use the ZUPT, it is necessary to detect zero velocity intervals reliably. Existing zero velocity intervals detection algorithms cannot provides good performance at high gait speeds or stair climbing. In this paper, we propose a novel zero velocity intervals detection approach based on sensors fusion. In this method, the measurements of accelerometer, gyroscope and pressure sensor are combined together to detect the zero velocity intervals. A new detector is derived based on the general likelihood ratio test (LRT) framework. The test results show that the new detector performs much better than the angular rate energy (ARE) detector.
\end{abstract}

Keywords: ZUPT; zero velocity intervals detection; sensors fusion; LRT.

\section{Introduction}

Self-contained inertial sensors have made pedestrian navigation an ideal way for positioning in emergency response missions in GNSS-denied environments. Among them, foot-mounted inertial measurement units (IMUs) have shown very promising visions [1-3]. The rapid development of micro-electromechanical systems (MEMS) has enabled the development of sensors-based inertial navigation system (INS) [4][5]. Unfortunately, these systems come error growth due to its integration nature. A widely used technique to suppress these errors in pedestrian navigation is known as the Zero-velocity-Updates (ZUPTs) aided Extended Kalman Filter (EKF) introduced in the literature [6]. During the pedestrian walking cycles, a foot periodically returns to a stationary state and keeps the state on the floor for a brief period. This interval is referred to as the zero velocity interval. The ZUPT method makes use of the fact that the sensor outputs will be governed only by sensor errors during the zero velocity interval, which can be used as the measurements fed into EKF to reduce the position error growth of the system. 
A range of detectors that intends to detect the zero-velocity phase have been proposed in [7-9]. However, these detectors are concluded in an ad-hoc manner, meanwhile the literature lacks a discussion of their characteristics. In [10], the angular rate energy (ARE) detector, the acceleration magnitude (MAG) detector, the acceleration moving variance (MV) detector, and the stance hypothesis optimal (SHOE) detector, are all summarized as generalized likelihood ratio tests. The detectors provide good performance at low gait speeds. In [11], an algorithm based on a hidden Markov model is constructed using a segmentation of gyroscope outputs. However, the state transition model is complex and not suitable for practical application.

In this paper, we propose a novel zero velocity intervals detection method based on sensors fusion. The measurements of accelerometer, gyroscope and pressure sensor are combined together to detect the zero velocity intervals. Then a new detector is derived based on the LRT framework. The new detector can provide good performance under running, stair climbing and stair descending conditions.

\section{Problem Description}

Assume that the inertial navigation system assembled with a pressure sensor and IMU (three-axis accelerometers and gyroscopes), has been attached into the heel of a foot. Let $\mathbf{m}_{k}$ denotes the output from the IMU and pressure sensor:

$$
\mathbf{m}_{k}=\left[\begin{array}{lll}
\mathbf{m}_{k}^{a} & \mathbf{m}_{k}^{\omega} & \mathbf{m}_{k}^{p}
\end{array}\right]^{T} .
$$

where $\mathbf{m}_{k}^{a}, \mathbf{m}_{k}^{\omega} \in \mathbb{R}^{3}$ are the acceleration and the angular rate vector, respective; $\mathbf{m}_{k}^{\rho} \in \mathbb{R}$ represents the measurement of the pressure sensor. The objective of the zero-velocity detection is to decide whether, during a time epoch consisting of $N$ observations between the time instants $n$ and $n+N-1$, the IMU is moving or stationary, given the measurement sequence $\mathbf{q}_{n}=\left\{\mathbf{m}_{k}\right\}_{k=n}^{n+N-1}$. Mathematically, the zero velocity detection problem can be formalized as a binary hypothesis test problem [10], where the detector can choose between the two hypotheses $H_{0}$ and $H_{1}$ defined as follows:

$$
\begin{aligned}
& H_{0}: \text { IMU is non-stationary } \\
& H_{1}: \text { IMU is stationary }
\end{aligned}
$$

The detector performance is then specified by the false-alarm $P_{\mathrm{FA}}=p\left(H_{1} \mid H_{0}\right)$ (the probability of deciding on the hypothesis $H_{1}$, when hypothesis $H_{0}$ is true) and the probability of detection $P_{D}=p\left(H_{0} \mid H_{1}\right)$ probability (the probability of deciding on the hypothesis $H_{0}$, when hypothesis 
$H_{1}$ is true). Depending on the Neyman-Pearson theorem, for a given $P_{\mathrm{FA}}$, we can choose between the two hypotheses to maximize $P_{D}$ when

$$
T\left(\mathbf{q}_{n}\right)=\frac{p\left(\mathbf{q}_{n} ; H_{1}\right)}{p\left(\mathbf{q}_{n} ; H_{0}\right)}>\gamma .
$$

\section{Measurement And Signal Model}

For IMU sensors, we restrict our analysis to the case that the IMU measurement can be described as follows:

$$
\boldsymbol{y}_{k}=\mathbf{s}_{k}+\mathbf{v}_{k} .
$$

where $\mathbf{s}_{k}=\left[\begin{array}{lll}\mathbf{s}_{k}^{a} & \mathbf{s}_{k}^{\omega} & \mathbf{s}_{k}^{\rho}\end{array}\right]^{T}, \mathbf{v}_{k}=\left[\begin{array}{lll}\mathbf{v}_{k}^{a} & \mathbf{v}_{k}^{\omega} & \mathbf{v}_{k}^{\rho}\end{array}\right]^{T}$. Here $\mathbf{s}_{k}^{a} \in \mathbb{R}^{3}$ and $\mathbf{s}_{k}^{\omega} \in \mathbb{R}^{3}$ are the acceleration and the angular rate vector, respectively at time $k ; \mathbf{s}_{k}^{\rho} \in \mathbb{R}$ denotes the output of pressure sensor. Moreover, $\mathbf{v}_{k}^{a} \in \mathbb{R}^{3}$ and $\mathbf{v}_{k}^{\omega} \in \mathbb{R}^{3}$ represent the measurement noise of the accelerometer and gyroscope, respectively; $\mathbf{v}_{k}^{\rho} \in \mathbb{R}$ is the measurement noise of the pressure sensor. Here, we assume that the components of $\mathbf{v}_{k}$ is independent identically distributed white Gaussian noise with covariance matrix:

$$
\mathbf{C}=\mathrm{E}\left\{\mathbf{v}_{k} \mathbf{v}_{k}^{T}\right\}=\left[\begin{array}{ccc}
\sigma_{a}^{2} \mathbf{I}_{3} & \mathbf{0}_{3} & \mathbf{0}_{3 \times 1} \\
\mathbf{0}_{3} & \sigma_{\omega}^{2} \mathbf{I}_{3} & \mathbf{0}_{3 \times 1} \\
\mathbf{0}_{1 \times 3} & \mathbf{0}_{1 \times 3} & \sigma_{\rho}^{2}
\end{array}\right]
$$

where $\sigma_{a}^{2}, \sigma_{\omega}^{2}$ and $\sigma_{\rho}^{2}$ are the noise variance of $s_{k}^{\theta}, s_{k}^{\varphi}$ and $s_{k}^{B}$, respectively. $\mathbf{I}_{3}\left(\mathbf{0}_{3}\right)$ denotes an identity(zero) matrix of three order, $\mathbf{0}_{3 \times 1}\left(\mathbf{0}_{1 \times 3}\right)$ denotes an zero matrix of size $3 \times 1(1 \times 3)$. E $\{\cdot\}$ denotes the expectation operation. When the IMU is stationary, the specific force measured by the accelerometer is only due to the known gravitation acceleration $g$, and the angular rate experienced by the IMU is zero. In addition, the output of the pressure senor will reach up to a maximum value $\rho_{\max }$ during this period. In order to unify the test criterion, we subtract $p_{\max }$ from the measurement of pressure sensor and select the absolute value instead:

$$
\mathbf{S}_{k}^{\rho^{\prime}}=\left|\mathbf{S}_{k}^{\rho}-\rho_{\max }\right|
$$

Then $\mathbf{s}_{k}^{\rho^{\prime}}$ is close to zero when the IMU is stationary. Hence, For the two hypotheses, the signal fulfills the restraint conditions :

$$
\begin{aligned}
& H_{0}: \exists k \in \Omega_{n}, \text { s.t. } \mathbf{s}_{k}^{a} \neq g \mathbf{u}_{n} \text { or } \mathbf{s}_{k}^{\omega} \neq \mathbf{0} \text { or } \mathbf{s}_{k}^{\rho^{\prime}} \neq 0 \\
& H_{1}: \forall k \in \Omega_{n}, \text { then } \mathbf{s}_{k}^{a}=g \mathbf{u}_{n} \text { and } \mathbf{s}_{k}^{\omega}=\mathbf{0} \text { and } \mathbf{s}_{k}^{\rho^{\prime}}=0 .
\end{aligned}
$$


where $\quad \mathbf{u}_{n} \in \mathbb{R}^{3},\left\|\mathbf{u}_{n}\right\|=1 \quad$ and $\quad \Omega_{n}=\{\ell \in \mathbb{N}: \mathrm{n} \leq \ell<N-1\}$.The unknown parameters of the signal for the two hypotheses are $\left\{\mathbf{s}_{k}\right\}_{k=n}^{n+N-1}$ and $\mathbf{u}_{n}$ respectively. The PDFs for the hypothesis $H_{i}(i=0,1)$ are given by:

$$
\begin{aligned}
p\left(\mathbf{q}_{n} ; \mathbf{s}_{k}, H_{i}\right) & =\prod_{k \in \Omega_{n}} p\left(\mathbf{y}_{k} ; \mathbf{s}_{k}, H_{i}\right) \\
= & \prod_{k \in \Omega_{n}} p\left(\mathbf{y}_{k}^{a} ; \mathbf{s}_{k}^{a}, H_{i}\right) p\left(\mathbf{y}_{k}^{\omega} ; \mathbf{s}_{k}^{\omega}, H_{i}\right) p\left(\mathbf{y}_{k}^{\rho} ; \mathbf{s}_{k}^{\rho}, H_{i}\right) .
\end{aligned}
$$

where

$$
\begin{aligned}
& p\left(\mathbf{y}_{k}^{a} ; \theta, H_{i}\right)=\frac{1}{\left(2 \pi \sigma_{a}^{2}\right)^{3 / 2}} \exp \left(-\frac{1}{2 \sigma_{a}^{2}}\left\|\mathbf{y}_{k}^{a}-\mathbf{s}_{k}^{a}(\theta)\right\|^{2}\right) \\
& p\left(\mathbf{y}_{k}^{\omega} ; \theta, H_{i}\right)=\frac{1}{\left(2 \pi \sigma_{\omega}^{2}\right)^{3 / 2}} \exp \left(-\frac{1}{2 \sigma_{\omega}^{2}}\left\|\mathbf{y}_{k}^{\omega}-\mathbf{s}_{k}^{\omega}(\theta)\right\|^{2}\right) \\
& p\left(\mathbf{y}_{k}^{\rho} ; \theta, H_{i}\right)=\frac{1}{\left(2 \pi \sigma_{\rho}^{2}\right)^{3 / 2}} \exp \left(-\frac{1}{2 \sigma_{\rho}^{2}}\left\|\mathbf{y}_{k}^{\rho}-\mathbf{s}_{k}^{\rho}(\theta)\right\|^{2}\right) .
\end{aligned}
$$

Under the hypothesis $H_{0}$, the unknown parameters in Eq. (9) are $\mathbf{s}_{k}$. Let $\hat{\mathbf{s}}_{k}$ be the MLE of $\mathbf{s}_{k}$, and depending on the theory of Maximum Likelihood Estimate for vector parameters, the MLEs $\hat{\mathbf{s}}_{k}$ can be obtained by:

$$
\left.\frac{\partial \ln p\left(\mathbf{q}_{n} ; \mathbf{s}_{k}, H_{0}\right)}{\partial \mathbf{s}_{k}}\right|_{\mathbf{s}_{k}=\hat{\mathbf{s}}_{k}}=\mathbf{0} .
$$

Then we can get:

$$
\hat{\mathbf{s}}_{k}^{a}=\frac{1}{N} \sum_{k \in \Omega_{n}} \mathbf{y}_{k}^{a}, \hat{\mathbf{s}}_{k}^{\omega}=\frac{1}{N} \sum_{k \in \Omega_{n}} \mathbf{y}_{k}^{\omega}, \hat{\mathbf{s}}_{k}^{\rho}=\frac{1}{N} \sum_{k \in \Omega_{n}} \mathbf{y}_{k}^{\rho} .
$$

Under the hypothesis $H_{1}, \mathbf{s}_{k}^{\omega}=\mathbf{0}, \mathbf{s}_{k}^{\rho^{\prime}}=0$, we can obtain the MLE of the unknown signal parameter $\mathbf{u}_{n}$ :

$$
\hat{\mathbf{u}}_{n}==\frac{\overline{\mathbf{y}}_{n}^{a}}{\left\|\overline{\mathbf{y}}_{n}^{a}\right\|}
$$

where

$$
\overline{\mathbf{y}}_{n}^{a}=\frac{1}{N} \sum_{k \in \Omega_{n}} \mathbf{y}_{k}^{a}
$$

If we combine Eq. (3) and Eq. (8), the GLRT becomes: 


$$
\begin{aligned}
T_{G}\left(\mathbf{q}_{n}\right) & =\exp \left(-\frac{1}{2 N \sigma_{\omega}^{2}}\left\|\sum_{k \in \Omega_{n}} \mathbf{y}_{k}^{\omega}\right\|^{2}\right) \cdot \exp \left(-\frac{1}{2 N \sigma_{\rho}^{2}}\left\|\sum_{k \in \Omega_{n}} \mathbf{y}_{k}^{\rho}\right\|^{2}\right) \\
& \cdot \exp \left(-\frac{1}{2 N \sigma_{a}^{2}}\left(N g-\left\|\sum_{k \in \Omega_{n}} \mathbf{y}_{k}^{a}\right\|\right)^{2}\right) .
\end{aligned}
$$

Taking the natural $\log$ on both sides and let $\gamma^{\prime}=-2 \ln \gamma$, then we can state the GLRT as: choose $H_{1}$ if

$$
\frac{1}{N}\left(\frac{1}{\sigma_{a}^{2}}\left(N g-\left\|\sum_{k \in \Omega_{n}} \mathbf{y}_{k}^{a}\right\|\right)^{2}+\frac{1}{\sigma_{\omega}^{2}}\left\|\sum_{k \in \Omega_{n}} \mathbf{y}_{k}^{\omega}\right\|^{2}+\frac{1}{\sigma_{\rho}^{2}}\left\|\sum_{k \in \Omega_{n}} \mathbf{y}_{k}^{\rho}\right\|^{2}\right)<\gamma^{\prime} .
$$

\section{Experiments}

Two tests were performed to assess the performance of the proposed method. The data is collected by a MIMU system as shown in Fig.1. The MIMU system is strapped into the heel of a shoe and the sampling rate is $500 \mathrm{~Hz}$.

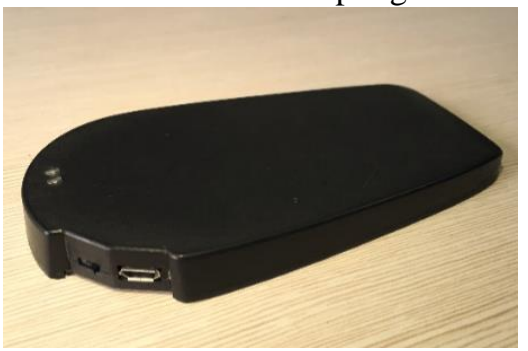

Fig.1. The MIMU system

In the first test, we walked along the corridors for one loop (approximately $5 \mathrm{~km} / \mathrm{h}$ ), then run along the same path (approximately $7 \mathrm{~km} / \mathrm{h}$ ). The total distance of the trajectory was approximately 320m. Fig. 2 shows the zero velocity detection results of the ARE method and our method during running. The blue denotes the angular rate energy. The red line represents the stationary state and moving state, and small values indicate the stationary state, large values indicate the moving state. It can be seen that the test statistic varies fast during pedestrian's motion. As illustrated by Fig.2, only four zero velocity intervals are detected by the ARE method during running. The proposed method performs much better and detects all the zero velocity intervals. 


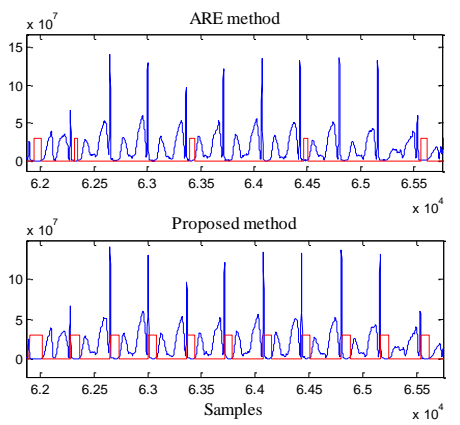

Fig.2. Zero velocity detection results

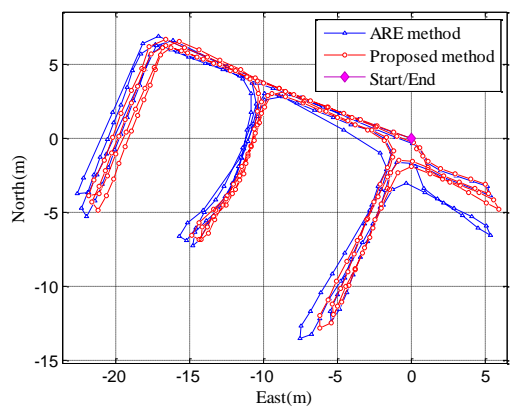

Fig.3. The trajectories of the first test

The trajectories of the ARE method and the proposed method are shown in Fig.3. It can be seen that the trajectories of the two method are similar during walking, but the difference is relatively large in the running stage. Our method achieves an error of no more than 0.6 meters. The ARE method has much larger positioning errors because some zero velocity intervals cannot be detected during running by this method..

In the second test, we walked from the first floor to the third floor, and then go back to the starting point. The X-Y plane trajectories are shown in Fig.4. The horizontal positioning error of the two method are similar which indicates that the performance of the ARE method and the proposed method is almost the same during walking in horizontal plane. Fig.5 shows the height trajectories of the two methods. It can be observed that the height of the proposed method is more accurate with height error no more than $0.2 \mathrm{~m}$. The height error of the ARE method is approximately $1 \mathrm{~m}$. The results indicate that the proposed method performs better during stair climbing and stair descending.

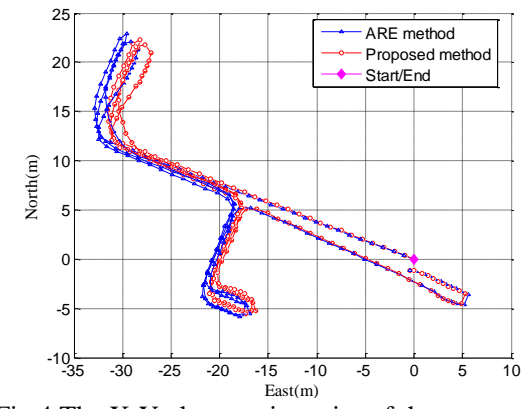

Fig.4.The X-Y plane trajectories of the test

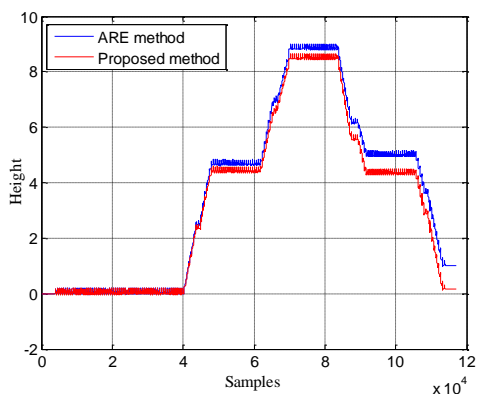

Fig.5.The height trajectories of the test

\section{Conclusion}

In this paper, we propose a novel zero velocity intervals detection method which can be used in indoor navigation systems. Existing methods usually work well 
for normal walking, but the detection is not reliable under running condition. Using the sensors fusion, the proposed method can provide good performance under walking, running, stair climbing and stair descending modes. The results of experiments on different motion modes have demonstrated the effectiveness and reliability of our approach.

\section{References}

1. S.Godha, G.Lachapelle, and M.E. Cannon, Integrated GPS/INS system for pedestrian navigation in a signal degraded environment, 19th International Technical Meeting of the Satellite Division, ION GNSS 2006.

2. L.Ojeda, and J.Borenstein, Personal dead-reckoning system for GPS-denied environments, 2007 IEEE International Workshop on Safety, Security and Rescue Robotics, SSRR 2007.

3. S. Wan, and E. Foxlin, Improved pedestrian navigation based on driftreduced MEMS IMU chip, International Technical Meeting 2010, ITM2010, 25-27th January, 2010.

4. I.Skog, J.O. Nilsson, and P.Händel, Pedestrian tracking using an IMU array, International Conference on Electronics, Computing and Communication Technologies, Bangalore, IN, 2014.

5. Y.Gu, Q.Song, Y.Li, and M.Ma, Foot-mounted Pedestrian Navigation based on Particle Filter with an Adaptive Weight Updating Strategy, The Journal of Navigation, 68(1), pp. 23-38, 2015.

6. E.Foxlin, Pedestrian tracking with shoe-mounted inertial sensors, IEEE Computer Graphics and Applications, vol. 25, no. 6, pp. 38-46, 2005.

7. S.P. Kwakkel, G.Lachapelle, and M.E. Cannon, GNSS aided in situ human lower limb kinematics during running, in Proc. of ION GNSS, (Savannah, GA,), Sept. 2008.

8. S.Godha, G.Lachapelle, and M.E. Cannon, Integrated GPS/INS system for pedestrian navigation in a signal degraded environment, in Proc. of ION GNSS, (Fort Worth, TX), Sept. 2006.

9. R.Feliz, E.Zalama, and J.G. Garcia-Bermejo, Pedestrian tracking using inertial sensors, Journal of Physical Agents, vol. 3, pp. 35-43,Jan. 2009.

10. I.Skog, J.O. Nilsson, and P. Händel, Zero-Velocity Detection-An Algorithm Evaluation, IEEE Transactions on Biomedical Engineering, 57(11), 2657-2666.

11. S.K Park and Y.S. Suh, A zero velocity detection algorithm using inertial sensors for pedestrian navigation systems. Sensors 2010, 10, 9163-9178. 